\title{
Assessing the effectiveness of NICE criteria for stratifying breast cancer risk in a UK cohort
}

\author{
Lucy A. Littlejohn ${ }^{1,2} \cdot \operatorname{Jim}$ Gibbs $^{3} \cdot$ Lee B. Jordan ${ }^{3} \cdot$ Zosia H. Miedzybrodzka $^{4} \cdot$ Christine Bell $^{4} \cdot$ David Goudie $^{2}$. \\ Jacqueline Dunlop ${ }^{2} \cdot$ Jonathan N. Berg ${ }^{1,2}$
}

Received: 24 July 2017 / Revised: 3 November 2017 / Accepted: 18 November 2017 / Published online: 15 January 2018

(c) European Society of Human Genetics 2018

\begin{abstract}
Breast cancer risk is a common indication for referral to clinical genetics services. UK National Institute of Health and Care Excellence (NICE) guidelines use family history (FH) to stratify by 10-year risk of breast cancer from age 40. Patients are stratified into population risk (PR, 10-year risk <3\%), moderate (MR, 3-8\%) and high risk (HR, $>8 \%$ ). Women at increased risk are offered screening at or prior to age 40 . To assess the clinical effectiveness of current risk stratification, FH data were obtained for all unaffected women with a FH of breast cancer aged $<50$, referred to cancer genetics from 2000-2010. Patients were risk stratified by NICE criteria, identifying patients who subsequently developed breast cancer. A total of 1409 women had 15,414 patient years of follow-up. Thirty invasive breast cancers developed, 13 in MR and 13 in HR women. Kaplan-Meier analysis demonstrated no significant difference in the rate of breast cancer development between PR and MR women from ages 40 to $49(\log$ rank $p=0.431)$. There was a significant difference between ages 40 and 49 years between PR and HR women $(p=0.036)$, but not on exclusion of BRCA mutation carriers $(p=0.136)$. NICE absolute 10-year risk thresholds between ages 40 and 49 were not met in any risk group, when risk was estimated using the guidelines ( $\mathrm{PR}=0.82 \%, \mathrm{MR}=1.68 \%, \mathrm{HR}=3.56 \%$ ). Our data suggest that improved criteria are required for risk assessment prior to age 50 and screening resources may be best focussed on those with highly penetrant mutations in cancer risk genes.
\end{abstract}

\section{Introduction}

Familial clustering of breast cancer is a common indication for referral to clinical genetics services. Whilst shared environmental factors contribute, they do not fully explain the risk, and genetic predisposition is thought to be a major factor. This can be due to rare, highly penetrant mutations, or multiple low penetrance variants [1, 2]. Risk assessment includes variant analysis for known cancer risk genes where appropriate, or assessment by family history (FH).

Lucy A. Littlejohn

lucy_littlejohn@outlook.com

1 Clinical Genetics, University of Dundee, Dundee, Angus, UK

2 Department of Clinical Genetics, Ninewells Hospital and Medical School, Dundee, Angus, UK

3 Department of Pathology, Ninewells Hospital and Medical School, Dundee, Angus, UK

4 Department of Clinical Genetics, Ashgrove House, Aberdeen, UK
The UK National Institute of Health and Care Excellence (NICE) provides guidance for classification and management of people with a FH of breast cancer (CG164) [3]. Patients are stratified according to FH into near population risk (PR), moderate risk (MR) and high risk (HR) based on percentage lifetime risk and 10-year risk from age 40. Risk stratification uses empirical criteria provided (shown in Table 1), or other models such as the Breast and Ovarian Analysis of Disease Incidence and Carrier Estimation Algorithm (BOADICEA), a computer program that is used to calculate the risks of breast and ovarian cancer in women based on their FH [4]. NICE recommends additional screening for women at MR and $\mathrm{HR}$, as seen in Table 1, in the form of mammograms or MRI. This is of relevance for younger women who are not yet enroled in the UK National Breast Screening Programme (NBSP), which offers 3-yearly mammograms to all women aged 50-70. To our knowledge, there has been no attempt to validate the empirical NICE criteria in women attending clinical genetics services regarding their breast cancer risk. 


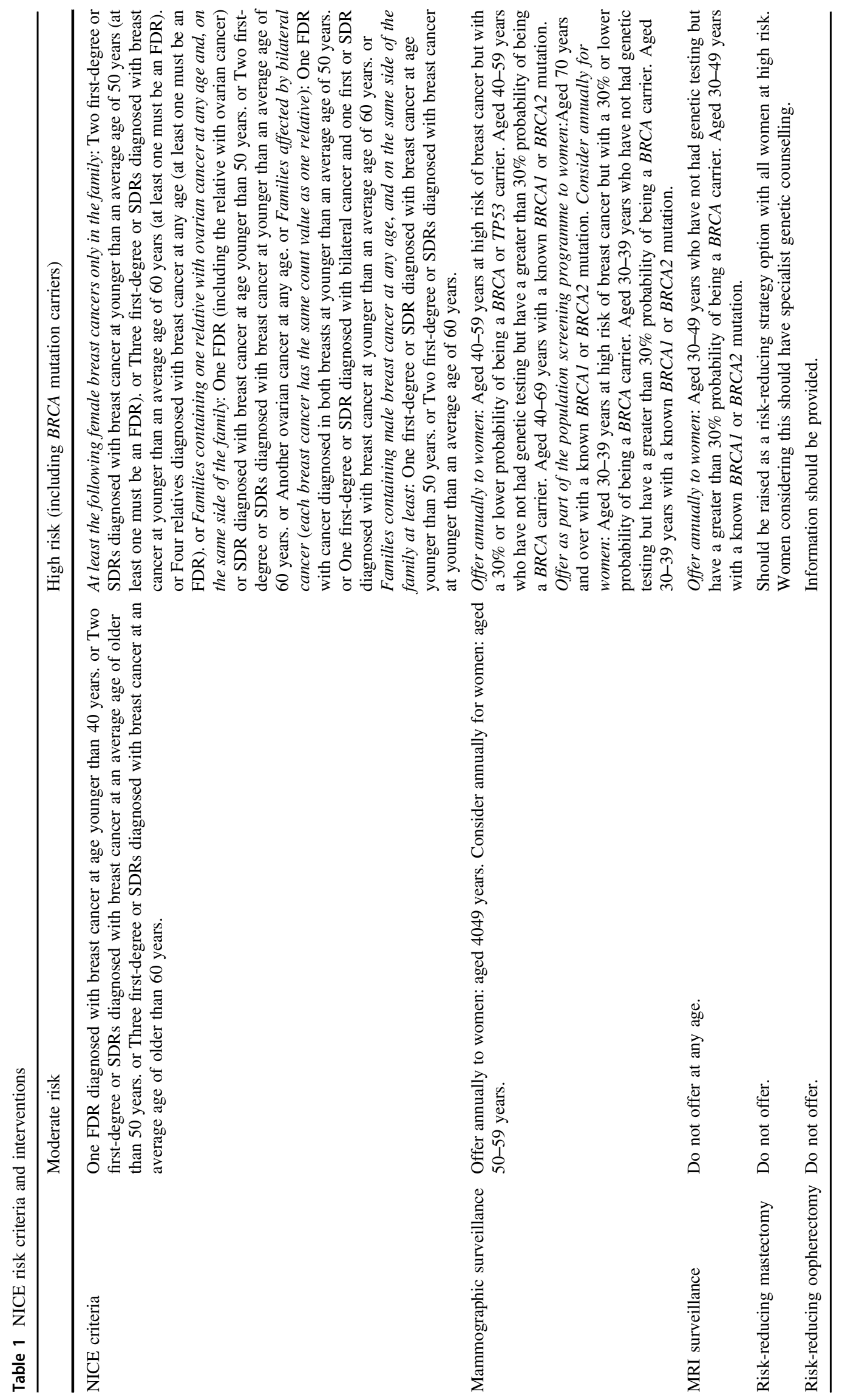


Table 2 Frequency and absolute risk of breast cancer by NICE risk category

\begin{tabular}{|c|c|c|c|c|}
\hline & \multicolumn{2}{|c|}{ Risk categorised using both sides of FH } & \multicolumn{2}{|c|}{ Risk categorised using only one side of FH } \\
\hline & \multicolumn{2}{|c|}{ Number of invasive cancers } & \multicolumn{2}{|c|}{ Number of invasive cancers } \\
\hline & \multicolumn{2}{|c|}{ \% 10-year absolute risk $(95 \% \mathrm{CI})$} & \multicolumn{2}{|c|}{$\%$ 10-year absolute risk $(95 \% \mathrm{CI})$} \\
\hline & 40-49 years & $50-59$ years & 40-49 years & $50-59$ years \\
\hline \multirow[t]{2}{*}{ Population risk } & 2 & 2 & 3 & 3 \\
\hline & $0.82 \%(0.72-0.94 \%)$ & $1.61 \%(1.42-1.83 \%)$ & $1.11 \%(0.10-1.23 \%)$ & $2.23 \%(2.02-2.47 \%)$ \\
\hline \multirow[t]{2}{*}{ Moderate risk } & 4 & 8 & 3 & 7 \\
\hline & $1.68 \%(1.53-1.83 \%)$ & $7.05 \%(6.78-7.31 \%)$ & $1.37 \%(1.23-1.52 \%)$ & $6.47 \%(6.19-6.75 \%)$ \\
\hline \multirow[t]{2}{*}{ High risk (excluding $B R C A$ carriers) } & 4 & 4 & 4 & 4 \\
\hline & $2.49 \%(2.28-2.70 \%)$ & $5.28 \%(4.93-5.64 \%)$ & $2.62 \%(2.40-2.84 \%)$ & $5.62 \%(5.26-5.99 \%)$ \\
\hline \multirow[t]{2}{*}{$B R C A$ carriers } & 2 & 1 & 2 & 1 \\
\hline & $26.67 \%(17.98-37.63 \%)$ & $52.63 \%(31.71-72.67 \%)$ & $26.67 \%(17.98-37.63 \%)$ & $52.63 \%(31.71-72.67 \%)$ \\
\hline \multirow[t]{2}{*}{ High risk (including $B R C A$ carriers) } & 6 & 5 & 6 & 5 \\
\hline & $3.56 \%(3.34-3.80 \%)$ & $6.44 \%(6.10-6.78 \%)$ & $3.74 \%(3.51-3.98 \%)$ & $6.84 \%(6.50-7.18 \%)$ \\
\hline
\end{tabular}

\section{Subjects and methods}

Female patients referred to clinical genetics services for breast cancer risk from 2000 to 2010 were included in the study. Patients were aged under 50 at initial consultation, with no personal history of breast and/or ovarian cancer. FH information was collected from clinical genetics services records. BRCA (referring to both BRCAI and BRCA2) mutation carriers were identified through the national BRCA testing service. Women who went on to develop breast cancer were identified by linkage to pathology records.

All women were risk categorised into PR, MR and $\mathrm{HR}$ as outlined in the NICE guidelines [3]. NICE guidelines do not state that affected relatives must be from the same side of the family. However, it is acknowledged that many clinicians interpret the guidelines this way. Therefore, all analyses were performed based on a risk categorisation which (1) did not assume and (2) assumed same-side FH as necessary to meet risk criteria. The result of BRCA testing was also considered for appropriate risk categorisation. This was time intensive with each case taking between 5-15 min for risk assignment. As this was done retrospectively using clinical notes, time taken for clinical consultation and confirmation of diagnoses of affected family members is not included.

Percentage 10-year risk was calculated for each risk category and for BRCA mutation carriers, for ages 40-49 and ages 50-59 years inclusive. Incidence of breast cancer per patient year of follow-up within each group was calculated, and extrapolated to give the 10-year absolute breast cancer risk. Kaplan-Meier Survival Analysis (KMSA) was used to assess the rate of breast cancer development across different risk categories and age ranges. Patients were censored at completed time of follow-up or at breast cancer diagnosis. The HR group was analysed both including and excluding BRCA carriers.

\section{Results}

In total, 1409 patients were eligible for inclusion with a total of 15,414 patient years of follow-up. Using both sides of the FH to calculate risk, 505 women were PR (35.8\%), $522 \mathrm{MR}$ (37\%) and $382 \mathrm{HR}(27.1 \%)$, including $12 \mathrm{BRCAl}$ and 10 $B R C A 2$ carriers. Using only a same-side $\mathrm{FH}$, there were 554 (39.3\%) PR, 490 (34.8\%) MR and 365 (25.9\%) HR women.

Thirty women developed an invasive cancer prior to May 2016. The frequency and percentage 10-year absolute risk are shown in Table 2. Not assuming a same-side FH, the highest absolute risk between the ages of 40 and 49 was in the HR group, both including (3.56\% (3.34-3.80\%) and excluding BRCA carriers $(2.49 \%$ (2.28-2.70\%). From ages 50 to 59 , the MR group had the highest percentage absolute risk, at $7.05 \%(6.78-7.31 \%)$.

Between ages 40 and 49, none of the groups met the 10year risk suggested by NICE guidelines. Assuming a sameside $\mathrm{FH}$, a similar pattern of absolute risk is seen, with no group reaching the screening threshold suggested by NICE.

Table 3 shows the results of KMSA. Not assuming sameside $\mathrm{FH}$, there is no significant difference in the rate of breast cancer development between the PR and MR groups from 40 to $49(p=0.431)$. A risk difference between these two groups emerges after the age of $50(p=0.037)$. When same-side FH is assumed, there is no significant difference in breast cancer rates between the PR and MR groups 
Table 3 Kaplan-Meier analysis of the rate of breast cancer diagnosis comparing NICE risk categories by age range

\begin{tabular}{|c|c|c|c|c|c|c|c|c|}
\hline & \multicolumn{4}{|c|}{ Same-side FH not assumed } & \multicolumn{4}{|c|}{ Same-side FH assumed } \\
\hline & \multicolumn{4}{|c|}{ KM Log-rank ( $p$-value) } & \multicolumn{4}{|c|}{ KM Log-rank ( $p$-value) } \\
\hline & $\begin{array}{l}\text { Total follow- } \\
\text { up time }\end{array}$ & $\begin{array}{l}<39 \\
\text { years }\end{array}$ & $\begin{array}{l}40-49 \\
\text { years }\end{array}$ & $\begin{array}{l}50-59 \\
\text { years }\end{array}$ & $\begin{array}{l}\text { Total follow- } \\
\text { up time }\end{array}$ & $\begin{array}{l}<39 \\
\text { years }\end{array}$ & $\begin{array}{l}40-49 \\
\text { years }\end{array}$ & $\begin{array}{l}50-59 \\
\text { years }\end{array}$ \\
\hline Population \& moderate & 0.048 & 0.341 & 0.431 & $\mathbf{0 . 0 3 7}$ & 0.134 & 0.283 & 0.791 & 0.11 \\
\hline Population \& high & 0.003 & 0.091 & 0.036 & 0.149 & 0.005 & 0.328 & 0.042 & 0.063 \\
\hline $\begin{array}{l}\text { Population \& high (BRCA } \\
\text { carriers excluded) }\end{array}$ & 0.019 & 0.085 & 0.136 & 0.145 & 0.027 & 0.317 & 0.171 & 0.131 \\
\hline Moderate \& high & 0.274 & 0.328 & 0.183 & 0.581 & 0.218 & 0.995 & 0.111 & 0.795 \\
\hline $\begin{array}{l}\text { Moderate \& high (BRCA carriers } \\
\text { excluded) }\end{array}$ & 0.644 & 0.299 & 0.499 & 0.598 & 0.505 & 0.963 & 0.334 & 0.942 \\
\hline Population \& moderate/high & 0.011 & 0.216 & 0.134 & 0.05 & 0.022 & 0.298 & 0.206 & 0.069 \\
\hline $\begin{array}{l}\text { Population \& moderate/high } \\
\text { (BRCA carriers excluded) }\end{array}$ & 0.024 & 0.217 & 0.241 & 0.049 & 0.049 & 0.292 & 0.383 & 0.093 \\
\hline
\end{tabular}

overall $(p=0.134)$ or across any age range $(<39$ years $p=$ $0.283,40-49$ years $p=0.791,50-59$ years $p=0.11)$.

Both not assuming and assuming same-side $\mathrm{FH}$, there is a difference in breast cancer rates between the PR and HR women from 40 to 49 ( $p=0.036$ and $p=0.042$, respectively). However, this significance is lost on exclusion of BRCA carriers ( $p=0.136$ and $p=0.171$, respectively). There is no significant difference in the rate of breast cancer between these groups from the ages of 50 to 59 not assuming or assuming same-side $\mathrm{FH}(p=0.149$ and $p=$ $0.063)$.

The MR and HR groups combined were compared with the PR group to try and detect a significantly increased rate of breast cancer in women deemed at any increased risk. Not assuming same-side FH, the MR/HR group (excluding BRCA carriers) had a significantly increased rate of breast cancer from 50 to 59 years $(p=0.049)$. There was no detectable difference in breast cancer rates between MR and HR women at any time.

\section{Discussion}

Before the age of 50, neither the MR nor HR groups have a risk that reached the suggested NICE 10-year threshold. KMSA showed the rate of breast cancer development under the age of 50 to be significantly greater for those with a BRCA mutation but, crucially, not for other MR or HR women in the cohort compared to the PR group.

Our study has used a real clinical cohort, based on routine clinical practice for patients referred over a 10-year period. In this context, empirical NICE risk criteria do not appear to achieve effective risk stratification of those without a highly penetrant mutation before the age of 50 . In the MR group, there was a detectable increase in cancer risk after the age of 50; however, additional screening is not mandated for this group. When interpreted as requiring a same-sided $\mathrm{FH}$, empirical criteria fail to detect this difference.

It is recognised that the moderately increased risk of breast cancer observed in some families may be due to a multifactorial, polygenic risk model. The greater ability of the guidance to identify at-risk women when both sides of a $\mathrm{FH}$ are used in risk estimation may reflect this model of inheritance, with risk alleles being transmitted from both sides of the family. Future routine clinical practice is likely to require the analysis of genetic variants contributing to polygenic risk to achieve better performing risk estimation models. This is currently under investigation [5, 6].

NICE guidelines do suggest the use of other methods of risk stratification, specifically BOADICEA [3]. There is evidence that other methods such as BOADICEA may be effective in risk stratification [7], although there is no direct published comparison with NICE empirical criteria.

This study has used a simple methodology to assess current clinical practice in UK cancer genetics. Of 1409 patients being screened over a 16-year period, 30 developed invasive breast cancer. In this cohort, the ability of the current guidance to identify at-risk women, once highly penetrant mutations are excluded, is poor. Though we have a moderate cohort size, we feel that these results are important and should encourage further investigation of the effectiveness of these national guidelines. It would appear beneficial to refine risk stratification methods to focus resources on women who will benefit most from early screening.

Acknowledgements We would like to acknowledge the recently retired Simon Ogston, statistician at the Department of Population Health Sciences, University of Dundee. 


\section{Compliance with Ethical Standards}

Conflict of interest The authors declare that they have no conflict of interest.

\section{References}

1. Milne RL, Burwinkel B, Michailidou K, et al. Common nonsynonymous SNPs associated with breast cancer susceptibility: findings from the Breast Cancer Association Consortium. Hum Mol Genet. 2014;23:6096-111.

2. Michailidou K, Beesley J, Lindstrom S, et al. Genome-wide association analysis of more than 120,000 individuals identifies 15 new susceptibility loci for breast cancer. Nat Genet. 2015;47:373-80.

3. National Institute for Health and Care Excellence. Familial breast cancer: classification, care and managing breast cancer and related risks in people with a family history of breast cancer CG164. NICE; 2013.

4. Antoniou AC, Pharoah PP, Smith P, Easton DF. The BOADICEA model of genetic susceptibility to breast and ovarian cancer. $\mathrm{Br} \mathrm{J}$ Cancer. 2004;91:1580-90.

5. Evans DG, Brentnall A, Byers H, et al. The impact of a panel of 18 SNPs on breast cancer risk in women attending a UK familial screening clinic: a case-control study. J Med Genet. 2017;54:111-3.

6. Kuchenbaecker KB, McGuffog L, Barrowdale D, et al. Evaluation of polygenic risk scores for Breast and Ovarian Cancer Risk Prediction in BRCA1 and BRCA2 mutation carriers. J Natl Cancer Inst. 2017. https://doi.org/10.1093/jnci/djw302.

7. Antoniou AC, Hardy R, Walker L, et al. Predicting the likelihood of carrying a BRCA1 or BRCA2 mutation: validation of BOADIECA, BRCAPRO, IBIS, Myriad and the Manchester scoring system using data from UK genetics clinics. J Med Genet. 2008;45:425-31. 